\title{
Tribonacci numbers with indices in arithmetic progression and their sums
}

\author{
Nurettin Irmak and Murat Alp
}




\title{
TRIBONACCI NUMBERS WITH INDICES IN ARITHMETIC PROGRESSION AND THEIR SUMS
}

\author{
NURETTIN IRMAK AND MURAT ALP
}

Received 3 May, 2012

\begin{abstract}
In this paper, we give a recurrence relation for the Tribonacci numbers with indices in aritmetics progression, $\left\{T_{r n+s}\right\}$ for $0 \leq s<n$. We find sums of $\left\{T_{r n}\right\}$ for arbitrary integer $r$ via matrix methods.
\end{abstract}

2000 Mathematics Subject Classification: 11B39; 11C20

Keywords: Tribonacci numbers, sums, matrix methods

\section{INTRODUCTION}

For $n \geq 3$, the Tribonacci numbers are defined by

$$
T_{n}=T_{n-1}+T_{n-2}+T_{n-3}
$$

with initial conditions $T_{0}=0, T_{1}=T_{2}=1$.

The Binet formula of the Tribonacci numbers is given by,

$$
\begin{aligned}
T_{n} & =c_{1} \alpha^{n+1}+c_{2} \beta^{n+1}+c_{3} \gamma^{n+1} \\
& =c_{4} \alpha^{n}+c_{5} \beta^{n}+c_{6} \gamma^{n}
\end{aligned}
$$

where $c_{1}=\frac{1}{(\alpha-\beta)(\alpha-\gamma)}, c_{2}=\frac{1}{(\beta-\alpha)(\beta-\gamma)}, c_{3}=\frac{1}{(\gamma-\alpha)(\gamma-\beta)}, c_{4}=\frac{1}{-\alpha^{2}+4 \alpha-1}, c_{5}=$ $\frac{1}{-\beta^{2}+4 \beta-1}, c_{6}=\frac{1}{-\gamma^{2}+4 \gamma-1}$ and $\alpha, \beta, \gamma$ are the roots of the equation $x^{3}-x^{2}-x-1=$ 0 . The Tribonacci sequence is a special generalization of Fibonacci sequence. Many authors studied the Tribonacci sequence and its various properties. For example, in [11], the author found a formula for the Tribonacci numbers by using analytic methods. In [13], the author studied relationships between third order sequences with different initial conditions. And in [10], the author investigated the Tribonacci numbers' De moivre-type identities. In [5], the author found some formulas and identities and generating matrices for the sequences $\left\{T_{n}\right\},\left\{T_{4 n}\right\}$ and their sums. In [9], one can find the well-known identities for Tribonacci sequences. For example, 
the generating matrix of Tribonacci sequence is given by,

$$
Q^{n}=\left[\begin{array}{lll}
1 & 1 & 1 \\
1 & 0 & 0 \\
0 & 1 & 0
\end{array}\right]^{n}=\left[\begin{array}{ccc}
T_{n+1} & T_{n}+T_{n-1} & T_{n} \\
T_{n} & T_{n-1}+T_{n-2} & T_{n-1} \\
T_{n-1} & T_{n-2}+T_{n-3} & T_{n-2}
\end{array}\right]
$$

For more details, we refer to $[1,2,11,12]$.

The matrix method is very useful method in order to obtain some identities for special sequences. For example, using matrix methods, the authors obtained some identities for various special sequences (see [3, 4, 6-8]) .

In this study, we will consider certain subsequences of the Tribonacci sequence of form $\left\{T_{r n+s}\right\}$ for arbitrary integers $r, s$ with $0 \leq s<r$. After, we will find some formulas for sums of the sequence $\left\{T_{r n}\right\}$ by using matrix methods.

Now we are giving a lemma and a theorem for the subsequence of the Tribonacci sequences to determine the recurrence relation.

Lemma 1. Let $\alpha, \beta$ and $\gamma$ are roots of $x^{3}-x^{2}-x-1=0$. Then, for any positive integer $r,\left(\alpha^{r}+\beta^{r}+\gamma^{r}\right),(\alpha \beta)^{r}+(\alpha \gamma)^{r}+(\beta \gamma)^{r}$ and $(\alpha \beta \gamma)^{r}$ are always integers.

Proof. We will use the induction method. Obviously, $\alpha+\beta+\gamma=1, \alpha \beta+\beta \gamma+$ $\alpha \gamma=-1$ and $\alpha \beta \gamma=1$. Now, suppose that $\left(\alpha^{n}+\beta^{n}+\gamma^{n}\right),(\alpha \beta)^{n}+(\alpha \gamma)^{n}+(\beta \gamma)^{n}$ and $(\alpha \beta \gamma)^{n}$ are the integers for $0<n \leq r$. Then,

$$
\begin{aligned}
\left(\alpha^{r}+\beta^{r}+\gamma^{r}\right)(\alpha+\beta+\gamma)= & \alpha^{r+1}+\beta^{r+1}+\gamma^{r+1}+\alpha^{r-1}(\alpha \beta+\alpha \gamma) \\
& +\beta^{r-1}(\alpha \beta+\beta \gamma)+\gamma^{r-1}(\alpha \gamma+\beta \gamma)
\end{aligned}
$$

and since $\alpha \beta+\beta \gamma+\alpha \gamma=-1$,

$$
\begin{aligned}
\left(\alpha^{r}+\beta^{r}+\gamma^{r}\right)(\alpha+\beta+\gamma)= & \alpha^{r+1}+\beta^{r+1}+\gamma^{r+1}+\alpha^{r-1}(-1-\beta \gamma) \\
& +\beta^{r-1}(-1-\alpha \gamma)+\gamma^{r-1}(-1-\alpha \beta) \\
= & \alpha^{r+1}+\beta^{r+1}+\gamma^{r+1}-\left(\alpha^{r-1}+\beta^{r-1}+\gamma^{r-1}\right) \\
& -(\alpha \beta \gamma)\left(\alpha^{r-2}+\beta^{r-2}+\gamma^{r-2}\right) .
\end{aligned}
$$

Finally, we obtain

$\alpha^{r+1}+\beta^{r+1}+\gamma^{r+1}=\alpha^{r}+\beta^{r}+\gamma^{r}+\alpha^{r-1}+\beta^{r-1}+\gamma^{r-1}+\alpha^{r-2}+\beta^{r-2}+\gamma^{r-2}$.

Since $\left(\alpha^{r}+\beta^{r}+\gamma^{r}\right),\left(\alpha^{r-1}+\beta^{r-1}+\gamma^{r-1}\right)$ and $\left(\alpha^{r-2}+\beta^{r-2}+\gamma^{r-2}\right)$ are the integers, $\alpha^{r+1}+\beta^{r+1}+\gamma^{r+1}$ is the integer. Similarly, it can be shown that the terms of $(\alpha \beta)^{r}+(\alpha \gamma)^{r}+(\beta \gamma)^{r}$ and $(\alpha \beta \gamma)^{r}$ are the integers.

Theorem 1. For $n>2$ and the integers $r$, such that $0 \leq s<r$,

$$
\begin{aligned}
T_{r n+s} & =\left(\alpha^{r}+\beta^{r}+\gamma^{r}\right) T_{r(n-1)+s}-\left((\alpha \beta)^{r}+(\alpha \gamma)^{r}+(\beta \gamma)^{r}\right) T_{r(n-2)+s} \\
& +(\alpha \beta \gamma)^{r} T_{r(n-3)+s},
\end{aligned}
$$

where $\alpha, \beta$ and $\gamma$ are the roots of characteristic polynomial of the Tribonacci sequence $x^{3}-x^{2}-x-1$. 
Proof. In order to prove the claim, we will use the Binet formula of the Tribonacci sequence. If we evaluate the right hand side of (1.2), then

$$
\begin{gathered}
\left(\alpha^{r}+\beta^{r}+\gamma^{r}\right) T_{r(n-1)+s} \\
-\left((\alpha \beta)^{r}+(\alpha \gamma)^{r}+(\beta \gamma)^{r}\right) T_{r(n-2)+s}+(\alpha \beta \gamma)^{r} T_{r(n-3)+s} \\
=\left(\alpha^{r}+\beta^{r}+\gamma^{r}\right)\left(c_{1} \alpha^{r n+s}+c_{2} \beta^{r n+s}+c_{3} \gamma^{r n+s}\right) \\
-\left((\alpha \beta)^{r}+(\alpha \gamma)^{r}+(\beta \gamma)^{r}\right)\left(c_{1} \alpha^{r(n-1)+s}+c_{2} \beta^{r(n-1)+s}+c_{3} \gamma^{r(n-1)+s}\right) \\
+(\alpha \beta \gamma)^{r}\left(c_{1} \alpha^{r(n-2)+s}+c_{2} \beta^{r(n-2)+s}+c_{3} \gamma^{r(n-2)+s}\right),
\end{gathered}
$$

where $c_{1}=\frac{1}{(\alpha-\beta)(\alpha-\gamma)}, c_{2}=\frac{1}{(\beta-\alpha)(\beta-\gamma)}$ and $c_{3}=\frac{1}{(\gamma-\alpha)(\gamma-\beta)}$. If we simplify the above equation, then we obtain

$$
\begin{gathered}
\left(\alpha^{r}+\beta^{r}+\gamma^{r}\right) T_{r(n-1)+s} \\
-\left((\alpha \beta)^{r}+(\alpha \gamma)^{r}+(\beta \gamma)^{r}\right) T_{r(n-2)+s}+(\alpha \beta \gamma)^{r} T_{r(n-3)+s} \\
=c_{1} \alpha^{r(n+1)}+c_{2} \beta^{r(n+1)}+c_{3} \gamma^{r(n+1)} \\
=T_{r n+s .}
\end{gathered}
$$

Thus the proof is complete.

When $r=1$ in the above theorem, we obtain the well-known Tribonacci recurrence.

\section{SUMS OF TERMS OF THE SUBSEQUENCE $\left\{T_{r n}\right\}$}

In this section, we will compute sums of the Tribonacci numbers $\left\{T_{r n}\right\}$ and their sums by matrix methods.

$$
S_{n, r}=\sum_{k=0}^{n} T_{r k}
$$

where $r$ is an integer, and define matrices $F_{r}$ and $G_{n, r}$ as shown,

$$
F_{r}=\left[\begin{array}{cccc}
1 & 0 & 0 & 0 \\
1 & C_{r}^{(1)} & -C_{r}^{(2)} & C_{r}^{(3)} \\
0 & 1 & 0 & 0 \\
0 & 0 & 1 & 0
\end{array}\right]
$$

and

$$
G_{n, r}=\left[\begin{array}{cccc}
T_{r}+T_{r}^{*} & 0 & 0 & 0 \\
S_{n, r} & T_{r(n+1)} & -C_{r}^{(2)} T_{r n}+C_{r}^{(3)} T_{r(n-1)} & C_{r}^{(3)} T_{r n} \\
S_{n-1, r} & T_{r n} & -C_{r}^{(2)} T_{r(n-1)}+C_{r}^{(3)} T_{r(n-2)} & C_{r}^{(3)} T_{r(n-1)} \\
S_{n-2, r} & T_{r(n-1)} & -C_{r}^{(2)} T_{r(n-2)}+C_{r}^{(3)} T_{r(n-3)} & C_{r}^{(3)} T_{r(n-2)}
\end{array}\right]
$$


where $C_{r}^{(1)}=\alpha^{r}+\beta^{r}+\gamma^{r}, C_{r}^{(2)}=(\alpha \beta)^{r}+(\beta \gamma)^{r}+(\alpha \gamma)^{r}$ and $C_{r}^{(3)}=(\alpha \beta \gamma)^{r}$. For $n \geq 3$, the sequence $\left\{T_{r}^{*}\right\}$ is defined as follows

$$
T_{n}^{*}=-T_{n-1}^{*}-T_{n-2}^{*}+T_{n-3},
$$

where initial conditions $T_{0}^{*}=T_{1}^{*}=0, T_{2}^{*}=1$.

Theorem 2. For $n \geq 3$,

$$
T_{r} F_{r}^{n}+T_{r}^{*} F_{r}^{n-1}=G_{n, r} .
$$

Proof. The proof follows from the induction method.

After some computations, the eigenvalues of matrix $F_{r}$ are $\alpha^{r}, \beta^{r}, \gamma^{r}$ and 1. Define two matrices $L_{r}$ and $W_{r}$ as follows

$$
L_{k}=\left[\begin{array}{cccc}
1 & 0 & 0 & 0 \\
0 & \alpha^{k} & 0 & 0 \\
0 & 0 & \beta^{k} & 0 \\
0 & 0 & 0 & \gamma^{k}
\end{array}\right] \text { and } W_{r}=\left[\begin{array}{cccc}
1 & 0 & 0 & 0 \\
\frac{-1}{C_{r}^{(1)}-C_{r}^{(2)}} & \alpha^{2 k} & \beta^{2 k} & \gamma^{2 k} \\
\frac{C_{r}^{(1)}-C_{r}^{(2)}}{C^{2}} & \alpha^{k} & \beta^{k} & \gamma^{k} \\
\frac{C_{r}^{(1)}-C_{r}^{(2)}}{1} & 1 & 1
\end{array}\right]
$$

Theorem 3. If $n>0$, then

$$
S_{n, r}=\frac{1}{C_{r}^{(1)}-C_{r}^{(2)}}\left\{T_{r(n+1)}-\left(C_{r}^{(2)}-1\right) T_{r n}+C_{r}^{(3)} T_{r(n-1)}-\left(T_{r}+T_{r}^{*}\right)\right\} .
$$

Proof. Since $\alpha, \beta$ are $\gamma$ different zero, then $\operatorname{det} L_{k} \neq 0$. One can check that

$$
T_{r} F_{r}^{n} W_{r}=T_{r} W_{r} L_{r}^{n}
$$

and

$$
T_{r}^{*} F_{r}^{n-1} W_{r}=T_{r}^{*} W_{r} L_{r}^{n-1} .
$$

If we sum both equations side by side, we obtain that

$$
\left(T_{r} F_{r}^{n}+T_{r}^{*} F_{r}^{n-1}\right) W_{r}=W_{r}\left(T_{r} L_{k}^{n}+T_{r}^{*} L_{k}^{n-1}\right) .
$$

By Theorem 2, we deduce

$$
G_{n, r} W_{r}=W_{r}\left(T_{r} L_{k}^{n}+T_{r}^{*} L_{k}^{n-1}\right)
$$

Equating the $(2,1)$ - elements of each sides of the above equation completes the proof.

In the above theorem, we give a formula for sum of the terms of the sequence $\left\{T_{r n}\right\}$ for arbitrary $r$ and for the generating matrix of the sums. Now we define two 
new matrices $K_{r}$ and $R_{n, r}$ in order to give a Binet like-formula for the sum of the terms of the sequence $\left\{T_{r n}\right\}$,

$$
K_{r}=\left[\begin{array}{cccc}
C_{r}^{(1)}+1 & -C_{r}^{(1)}-C_{r}^{(2)} & C_{r}^{(2)}+1 & -1 \\
1 & 0 & 0 & 0 \\
0 & 1 & 0 & 0 \\
0 & 0 & 1 & 0
\end{array}\right]
$$

and

where

$$
R_{n, r}=\left[\begin{array}{cccc}
S_{n+1, r} & X_{n, r} & Y_{n, r} & -S_{n, r} \\
S_{n, r} & X_{n-1, r} & Y_{n-1, r} & -S_{n-1, r} \\
S_{n-1, r} & X_{n-2, r} & Y_{n-2, r} & -S_{n-2, r} \\
S_{n-2, r} & X_{n-3, r} & Y_{n-3, r} & -S_{n-3, r}
\end{array}\right]
$$

$$
\begin{gathered}
X_{n, r}=-\left(C_{r}^{(1)}+C_{r}^{(2)}\right) S_{n, r}+\left(C_{r}^{(2)}+1\right) S_{n-1, r}-S_{n-2, r}, \\
Y_{n}=\left(C_{r}^{(2)}+1\right) S_{n, r}+S_{n-1, r},
\end{gathered}
$$

and $S_{n, r}$ was defined by (2.1).

Theorem 4. For $n \geq 3$,

$$
T_{r} K_{r}^{n}+T_{r}^{*} K_{r}^{n-1}=R_{n, r}
$$

where $\left\{T_{r}\right\}$ is the Tribonacci sequence and the sequence $\left\{T_{r}^{*}\right\}$ is defined as in (2.2).

Proof. The proof follows from the induction method.

Now we define two matrices $\Delta_{1}$ and $D_{r}$ as follow

$$
\Delta_{1}=\left[\begin{array}{cccc}
\alpha^{3 r} & \beta^{3 r} & \gamma^{3 r} & 1 \\
\alpha^{2 r} & \beta^{2 r} & \gamma^{2 r} & 1 \\
\alpha^{r} & \beta^{r} & \gamma^{r} & 1 \\
1 & 1 & 1 & 1
\end{array}\right] \text { and } D_{r}=\left[\begin{array}{cccc}
\alpha^{r} & 0 & 0 & 0 \\
0 & \beta^{r} & 0 & 0 \\
0 & 0 & \gamma^{r} & 0 \\
0 & 0 & 0 & 1
\end{array}\right] .
$$

Theorem 5. For $n>4$,

$$
\begin{array}{r}
S_{n, r}=T_{r}\left(\frac{\alpha^{r(n+2)}}{\left(\alpha^{r}-1\right)\left(\alpha^{r}-\beta^{r}\right)\left(\alpha^{r}-\gamma^{r}\right)}+\frac{\beta^{r(n+2)}}{\left(\beta^{r}-1\right)\left(\beta^{r}-\alpha^{r}\right)\left(\beta^{r}-\gamma^{r}\right)}\right. \\
\left.+\frac{\gamma^{r(n+2)}}{\left(\gamma^{r}-1\right)\left(\gamma^{r}-\alpha^{r}\right)\left(\gamma^{r}-\beta^{r}\right)}\right)+ \\
T_{r}^{*}\left(\frac{\alpha^{r(n+1)}}{\left(\alpha^{r}-1\right)\left(\alpha^{r}-\beta^{r}\right)\left(\alpha^{r}-\gamma^{r}\right)}+\frac{\beta^{r(n+1)}}{\left(\beta^{r}-1\right)\left(\beta^{r}-\alpha^{r}\right)\left(\beta^{r}-\gamma^{r}\right)}\right. \\
\left.+\frac{\gamma^{r(n+1)}}{\left(\gamma^{r}-1\right)\left(\gamma^{r}-\alpha^{r}\right)\left(\gamma^{r}-\beta^{r}\right)}\right) .
\end{array}
$$


Proof. The characteristic equation of the matrix $K_{r}$ is

$$
x^{4}-\left(C_{r}^{(1)}+1\right) x^{3}+\left(C_{r}^{(1)}+C_{r}^{(2)}\right) x^{2}-\left(C_{r}^{(1)}+1\right) x+1=0 .
$$

Computing roots of the equation, we obtain $\alpha^{r}, \beta^{r}, \gamma^{r}$ and 1. So the matrix $K_{r}$ is diagonalizable. It can be shown that

$$
T_{r} K_{r}^{n} \Delta_{1}=T_{r} \Delta_{1} D_{r}^{n}
$$

and

$$
T_{r}^{*} K_{r}^{n-1} \Delta_{1}=T_{r}^{*} \Delta_{1} D_{r}^{n-1} .
$$

If we sum the both equalities, we obtain

$$
\left(T_{r} K_{r}^{n}+T_{r}^{*} K_{r}^{n-1}\right) \Delta_{1}=\Delta_{1}\left(T_{r} D_{r}^{n}+T_{r}^{*} D_{r}^{n-1}\right) .
$$

Using (2.3), we deduce that

$$
R_{n, r} \Delta_{1}=\Delta_{1}\left(T_{r} D_{r}^{n}+T_{r}^{*} D_{r}^{n-1}\right) .
$$

So, we have the following equation system,

$$
\begin{gathered}
\alpha^{3 r} x(r)_{i, 1}+\alpha^{2 r} x(r)_{i, 2}+\alpha^{r} x(r)_{i, 3}+x(r)_{i, 4}=T_{r} \alpha^{r(n-i)+4 r}+T_{r}^{*} \alpha^{r(n-i-1)+4 r} \\
\beta^{3 r} x(r)_{i, 1}+\beta^{2 r} x(r)_{i, 2}+\beta^{r} x(r)_{i, 3}+x(r)_{i, 4}=T_{r} \beta^{r(n-i)+4 r}+T_{r}^{*} \beta^{r(n-i-1)+4 r} \\
\gamma^{3 r} x(r)_{i, 1}+\gamma^{2 r} x(r)_{i, 2}+\gamma^{r} x(r)_{i, 3}+x(r)_{i, 4}=T_{r} \gamma^{r(n-i)+4 r}+T_{r}^{*} \gamma^{r(n-i-1)+4 r} \\
x(r)_{i, 1}+x(r)_{i, 2}+x(r)_{i, 3}+x(r)_{i, 4}=T_{r}+T_{r}^{*}
\end{gathered}
$$

where $R_{n, r}=\left[x(r)_{i, j}\right]$. In order to obtain $x(r)_{2,1}$, we use Cramer's method

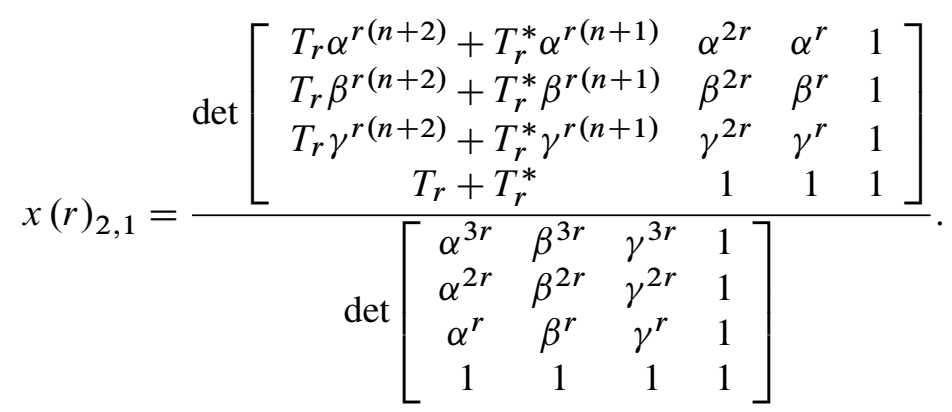

If we expand the first column of the matrix,

$$
\operatorname{det}\left[\begin{array}{cccc}
T_{r} \alpha^{r(n+2)}+T_{r}^{*} \alpha^{r(n+1)} & \alpha^{2 r} & \alpha^{r} & 1 \\
T_{r} \beta^{r(n+2)}+T_{r}^{*} \beta^{r(n+1)} & \beta^{2 r} & \beta^{r} & 1 \\
T_{r} \gamma^{r(n+2)}+T_{r}^{*} \gamma^{r(n+1)} & \gamma^{2 r} & \gamma^{r} & 1 \\
T_{r}+T_{r}^{*} & 1 & 1 & 1
\end{array}\right]
$$




$$
=\operatorname{det}\left[\begin{array}{cccc}
T_{r} \alpha^{r(n+2)} & \alpha^{2 r} & \alpha^{r} & 1 \\
T_{r} \beta^{r(n+2)} & \beta^{2 r} & \beta^{r} & 1 \\
T_{r} \gamma^{r(n+2)} & \gamma^{2 r} & \gamma^{r} & 1 \\
T_{r} & 1 & 1 & 1
\end{array}\right]+\operatorname{det}\left[\begin{array}{cccc}
T_{r}^{*} \alpha^{r(n+1)} & \alpha^{2 r} & \alpha^{r} & 1 \\
T_{r}^{*} \beta^{r(n+1)} & \beta^{2 r} & \beta^{r} & 1 \\
T_{r}^{*} \gamma^{r(n+1)} & \gamma^{2 r} & \gamma^{r} & 1 \\
T_{r}^{*} & 1 & 1 & 1
\end{array}\right],
$$

and after some simplifications, we obtain $S_{n, r}$ as

$$
\begin{gathered}
=T_{r}\left(\frac{\alpha^{r(n+2)}}{\left(\alpha^{r}-1\right)\left(\alpha^{r}-\beta^{r}\right)\left(\alpha^{r}-\gamma^{r}\right)}+\frac{\beta_{n, r}}{\left(\beta^{r}-1\right)\left(\beta^{r}-\alpha^{r}\right)\left(\beta^{r}-\gamma^{r}\right)}\right. \\
\left.+\frac{\gamma^{r(n+2)}}{\left(\gamma^{r}-1\right)\left(\gamma^{r}-\alpha^{r}\right)\left(\gamma^{r}-\beta^{r}\right)}\right)+ \\
T_{r}^{*}\left(\frac{\alpha^{r(n+1)}}{\left(\alpha^{r}-1\right)\left(\alpha^{r}-\beta^{r}\right)\left(\alpha^{r}-\gamma^{r}\right)}+\frac{\beta^{r(n+1)}}{\left(\beta^{r}-1\right)\left(\beta^{r}-\alpha^{r}\right)\left(\beta^{r}-\gamma^{r}\right)}\right. \\
\left.+\frac{\gamma^{r(n+1)}}{\left(\gamma^{r}-1\right)\left(\gamma^{r}-\alpha^{r}\right)\left(\gamma^{r}-\beta^{r}\right)}\right) .
\end{gathered}
$$

\subsection{Determinantal representations}

In this section, we give some relationships between the sequence $\left\{T_{r n}\right\}$, the sum of terms of the sequence and the permanents of certain matrices. We define $n \times n$ matrices $H_{n, r}, Z_{n, r}$ as follows

$$
H_{n, r}=\left[u(r)_{i, j}\right]= \begin{cases}C_{r}^{(1)} & \text { if } i=j \\ -C_{r}^{(2)} & \text { if } j=i+1 \\ C_{r}^{(3)} & \text { if } j=i+2 \\ 1 & \text { if } i=j+1 \\ 0 & \text { otherwise }\end{cases}
$$

and

$$
Z_{n, r}=\left[\begin{array}{ccccc}
1 & 1 & & \cdots & 1 \\
1 & & & \\
0 & & H_{n-1} & & \\
\vdots & & & \\
0 & & &
\end{array}\right]
$$

We present some relationships with the following theorem.

Theorem 6. For $n>1$

(i) $T_{r}$ per $H_{n, r}+T_{r}^{*}$ per $H_{n-1, r}=T_{r(n+1)}$

(ii) $T_{r}$ per $Z_{n, r}+T_{r}^{*}$ per $Z_{n-1, r}=\sum_{j=1}^{n} T_{r j}$ 
Proof. (i) If we expand the permanent of matrix $H_{n, r}$ according to the first column, then we obtain

$$
\operatorname{perH}_{n+1, r}=C_{r}^{(1)} \operatorname{perH}_{n, r}-C_{r}^{(2)} \operatorname{perH}_{n-1, r}+C_{r}^{(3)} \operatorname{perH}_{n-1, r} .
$$

Since $\operatorname{per}_{n, r}$ and $\left\{T_{r n}\right\}$ have the same recurrence relation and initial conditions, the proof is complete.

(ii) It can be proven similarly to the first identity.

\section{COMBINATORIAL REPRESENTATIONS}

In this part, we give some combinatorial representations of the terms of the sum of subsequence of the Tribonacci sequence and the entries of the $n^{\text {th }}$ power of the companion matrix

$$
A_{k}\left(k_{1}, k_{2}, \ldots, k_{k}\right)=\left[\begin{array}{cccc}
k_{1} & k_{2} & \cdots & k_{k} \\
1 & 0 & \cdots & 0 \\
\vdots & \ddots & \ddots & \ddots \\
0 & 0 & 1 & 0
\end{array}\right]
$$

In [2], we can see the following results;

Theorem 7. The $(i, j)$ entry $a_{i, j}^{(n)}\left(k_{1}, k_{2}, \ldots, k_{k}\right)$ in matrix $A_{k}^{(n)}\left(k_{1}, k_{2}, \ldots, k_{k}\right)$ is given by following formula:

$$
\begin{gathered}
a_{i, j}^{(n)}\left(k_{1}, k_{2}, \ldots, k_{k}\right) \\
=\sum_{\left(t_{1}, t_{2}, \ldots, t_{k}\right)} \frac{t_{j}+t_{j+1}+\cdots+t_{k}}{t_{1}+t_{2}+\cdots+t_{k}} \times\left(\begin{array}{c}
t_{1}+t_{2}+\cdots+t_{k} \\
t_{1}, t_{2}, \ldots, t_{k}
\end{array}\right) k_{1}^{t_{1}} \ldots k_{k}^{t_{k}}
\end{gathered}
$$

where the summation is over nonnegative integers satisfying $t_{1}+2 t_{2}+\cdots+k t_{k}=$ $n-i+j$, and the coefficient in (3.1) is defined to be 1 if $n=i-j$.

Corollary 1. Let $S_{n, r}$ be the sum of Tribonacci numbers where the subscripts form an arithmetic sequence. Then

$$
\begin{aligned}
S_{n, r}=\sum_{\left(r_{1}, r_{2}, r_{3}, r_{4}\right)}\left(\begin{array}{c}
r_{1}+r_{2}+r_{3}+r_{4} \\
r_{1}, r_{2}, r_{3}, r_{4}
\end{array}\right)\left(C_{r}^{(1)}\right. & +1)^{r_{1}}\left(-C_{r}^{(1)}+C_{r}^{(2)}\right)^{r_{2}} \\
& \times\left(-C_{r}^{(2)}+1\right)^{r_{3}}(-1)^{r_{4}}
\end{aligned}
$$

where the summation is over nonnegative integers satisfying $r_{1}+2 r_{2}+3 r_{3}+4 r_{4}=$ $n-1$.

Corollary 2. Let $T_{n}$ be the $n^{\text {th }}$ Tribonacci number. Then

$$
T_{r n}=\sum_{\left(t_{1}, t_{2}, t_{3}\right)}\left(\begin{array}{c}
t_{1}+t_{2}+t_{3} \\
t_{1}, t_{2}, t_{3}
\end{array}\right)\left(C_{r}^{(1)}\right)^{t_{1}}\left(-C_{r}^{(2)}\right)^{t_{2}}\left(C_{r}^{(3)}\right)^{t_{3}}
$$


where the summation is over nonnegative integers satisfying $t_{1}+2 t_{2}+3 t_{3}=n-1$.

\section{ACKNOWLEDGEMENT}

The authors would like to thank the anonymous referee for a number of helpful suggestions.

\section{REFERENCES}

[1] K. Alladi and V. E. j. Hoggatt, "On Tribonacci numbers and related functions," Fibonacci Q., vol. 15 , pp. 42-45, 1977.

[2] W. Y. C. Chen and J. D. Louck, "The combinatorial power of the companion matrix," Linear Algebra Appl., vol. 232, pp. 261-278, 1996.

[3] E. Kiliç, "The generalized order- $k$ Fibonacci-Pell sequence by matrix methods," J. Comput. Appl. Math., vol. 209, no. 2, pp. 133-145, 2007.

[4] E. Kiliç, "Sums of generalized Fibonacci numbers by matrix methods," Ars Comb., vol. 84, pp. 23-31, 2007.

[5] E. Kiliç, "Tribonacci sequences with certain indices and their sums," Ars Comb., vol. 86, pp. 13-22, 2008.

[6] E. Kiliç, "The generalized Fibonomial matrix," Eur. J. Comb., vol. 31, no. 1, pp. 193-209, 2010.

[7] E. Kiliç, "A matrix approach for generalizing two curious divisibility properties," Math. Notes, Miskolc, vol. 13, no. 2, pp. 389-396, 2012.

[8] E. Kiliç and P. Stănică, "A matrix approach for general higher order linear recurrences," Bull. Malays. Math. Sci. Soc. (2), vol. 34, no. 1, pp. 51-67, 2011.

[9] T. Koshy, Fibonacci and Lucas numbers with applications, ser. Pure and Applied Mathematics. A Wiley-Interscience Series of Texts, Monographs, and Tracts. New York: Wiley, 2001.

[10] P.-Y. Lin, "De Moivre-type identities for the Tribonacci numbers," Fibonacci Q., vol. 26, no. 2, pp. 131-134, 1988.

[11] C. P. McCarty, "A formula for Tribonacci numbers," Fibonacci Q., vol. 19, pp. 391-393, 1981.

[12] H. Minc, "Permanent of (0,1)-circulants," Can. Math. Bull., vol. 7, pp. 253-263, 1964.

[13] S. Pethe, "Some identities for Tribonacci sequences," Fibonacci Q., vol. 26, no. 2, pp. 144-151, 1988.

\section{Authors' addresses}

\section{Nurettin Irmak}

Niğde University, Mathematics Department, 51240 Niğde, TURKEY

E-mail address: nirmakenigde.edu.tr

\section{Murat Alp}

Niğde University, Mathematics Department, 51240 Niğde, TURKEY

E-mail address: muratalp@nigde.edu.tr 